\title{
Circumferential Fusion through All-Posterior Approach in Andersson Lesion
}

\author{
Sreekanth Reddy Rajoli, Rishi Mugesh Kanna, Siddharth N. Aiyer, \\ Ajoy Prasad Shetty, Shanmuganathan Rajasekaran \\ Department of Orthopaedics and Spine Surgery, Ganga Hospital, Coimbatore, India
}

\begin{abstract}
Study Design: Retrospective case series.
Purpose: To assess safety and efficacy of single stage, posterior stabilisation and anterior cage reconstruction through the transforaminal or lateral extra-cavitary route for Andersson lesions.

Overview of Literature: Pseudoarthrosis in ankylosing spondylitis (Andersson lesion, AL) can cause progressive kyphosis and neurological deficit. Management involves early recognition and surgical stabilisation in patients with instability. However, the need and safety of anterior reconstruction of the vertebral body defect remains unclear.

Methods: Twenty consecutive patients with AL whom presented with instability back pain and or neurological deficit were managed by single stage posterior approach with long segment pedicle screw fixation and anterior vertebral reconstruction. Radiological evaluation included- the regional kyphotic angle, measurement of anterior defect in computed tomography (CT) scan and the spinal cord status in magnetic resonance imaging. Radiological outcomes were assessed for fusion and kyphosis correction. Functional outcomes were assessed with visual analogue scale (VAS), ankylosing spondylitis quality of life (ASQoL) and Oswestry disability index (ODI). Results: The mean age of the patients was 50.1 years (male, 18; female, 2). The levels affected include thoracolumbar ( $n=12)$, lower thoracic $(n=5)$ and lumbar $(n=3)$ regions. The mean level of fixation was $6.2 \pm 2.4$ vertebrae. The mean anterior column defect was $1.6 \pm 0.6$ $\mathrm{cm}$. The mean surgical duration, blood loss and hospital stay were 112 minutes, $452 \mathrm{~mL}$ and 6.2 days, respectively. The mean followup was 2.1 years. At final follow up, VAS for back pain improved from 8.2 to 2.4 while ODI improved from 62.7 to $18.5(p<0.05)$ and ASQoL improved from $14.3 \pm 2.08$ to $7.90 \pm 1.48(p<0.05)$. All patients had achieved radiological union at a mean $7.2 \pm 4.6$ months. The mean regional kyphotic angle was $27^{\circ}$ preoperatively, $16.7^{\circ}$ postoperatively and $18.1^{\circ}$ at the final follow-up.

Conclusions: Posterior stabilisation and anterior reconstruction with cage through an all-posterior approach is safe and can achieve good results in Andersson lesions.
\end{abstract}

Keywords: Andersson lesion; Ankylosing spondylitis; pseudoarthrosis; all-posterior approach; kyphosis

\section{Introduction}

Andersson lesion (AL) is a non-infective, inflammatory lesion of the fused disc or vertebra in patients with late stage ankylosing spondylitis (AS). From the time, Andersson [1] first described these lesions, many etiologies have been proposed for AL including inflammation, trauma and infection [2-6]. Though it is commonly misdiagnosed

Received Oct 18, 2016; Revised Nov 11, 2016; Accepted Nov 28, 2016

Corresponding author: S. Rajasekaran

Department of Orthopaedics, Traumatology and Spine Surgery, Ganga Hospital,

313, Mettupalayam road, Coimbatore 641043, India

Tel: +91-422-2485000, Fax: +91-422-2451444, E-mail: rajasekaran.orth@gmail.com 
as infective spondylodiscitis, an infective etiology has not been consistently proven. However, Lohr et al. [6] in their study isolated Staphylococcus aureus from a patient of AS who presented with a lesion similar to AL. Nikolaisen and Nossent [3] observed that inflammation could be the main etiology for AL in their study. Unrecognised trauma is also a possible etiology, since several studies have shown that at least $40 \%-60 \%$ of patients do recognise a previous trauma [6-8]. Despite many possible etiologies, the final common result is pseudoarthrosis involving either one or both spinal columns resulting in spinal instability [7].

Surgical stabilisation is the mainstay of treatment especially in those with chronic axial pain, neurological deficit and kyphosis but it is unclear whether stabilisation of the spine alone suffices or is there a need to debride and support the anterior column. Similarly the surgical methods described have been versatile including anterior surgery with fusion, combined anterior and posterior surgery, posterior short segment fixation and posterior long segment fixation with anterior reconstruction [8-13]. Anterior only surgery was performed initially but did not achieve popularity because of risks of pseudoarthrosis, higher morbidity associated with anterior surgery, difficulties in reaching the lesion in the kyphotic spine and compromised lung function in AS patients $[8,14,15]$. Though posterior surgery is commonly done because of its ease and familiarity, it is associated with disadvantages such as extensive muscle dissection leading to increased blood loss, risk of implant failure due to osteoporosis in the ankylosed spine and the primary anterior lesion remains unattended [16]. Some authors have performed combined anterior-posterior surgeries, which is a major undertaking in AS patients with compromised lung function $[9,10,17]$. An all-posterior approach, where the anterior lesion is reached from posterior, through a window in the transforaminal region, or a transpedicular or lateral extra-cavitary route, not only avoids the complications of the anterior approach but also provides the required biomechanical support $[12,13]$. However, till now no single surgical option is considered ideal. Due to the rarity of the lesion, the literature is ambiguous regarding the type of surgical approach, extent of fixation and the need for debridement and anterior reconstruction. The purpose of this study is to evaluate the safety, efficacy and feasibility of a posterior alone surgery and anterior reconstruction through a transforaminal or lateral extra-cavitary access in patients with $\mathrm{AL}$.

\section{Materials and Methods}

The study was approved by the Institutional Review Board of Ganga Medical Centre and Hospitals, Coimbatore (IRB Registration no. 16-S-9). From January 2009 to April 2014, 20 consecutive patients diagnosed as ankylosing spondylitis with Andersson lesion, who underwent surgery, were included in the study and retrospectively evaluated based on case records. Those with acute fractures, and early lesions involving only the anterior column were not included in the study. The indications for surgery were persistent back pain $(n=16)$, neurological deficit $(n=3)$, and progressive kyphosis $(\mathrm{n}=1)$.

All the patients were radiologically assessed preoperatively by means of plain radiographs, CT scan and magnetic resonance imaging (MRI). In the lateral radiographs, the regional kyphosis was measured using the standard Cobb's method before and after surgery (Fig. 1). A line was drawn along the upper endplate of cranial intact vertebra and the lower endplate of the vertebra one level caudal to the lesion and the angle subtended between the two lines provides the Cobb angle. CT scan provided the extent of vertebral destruction and the mid-coronal section through the middle of the vertebral body was used to measure the bony defect of the pseudoarthrosis. The axial and sagittal MRI sections were utilised to evaluate any compression of the spinal cord.

The functional outcomes were evaluated by visual analogue score (VAS) for axial pain and ankylosing spondylitis quality of life (ASQOL) and Oswestry disability index (ODI) through self-administered patient questionnaires before surgery and at follow up. Neurological status was assessed by means of American Spine Injury Association (ASIA) grading.

\section{Surgical technique}

All the surgeries were performed entirely by the posterior approach by a single surgical team. Under general anaesthesia, the patient was placed in prone position with due care to protect the stability of the spine. To minimise intra-operative blood loss, Tranexamic acid $10 \mathrm{mg} / \mathrm{kg}$ intravenous infusion and hypotensive anaesthesia (mean arterial blood pressure $<90 \mathrm{~mm} \mathrm{Hg}$ ) was used. The spine was exposed using the standard posterior midline approach. The exposure was performed laterally up to the costotransverse joint in the thoracic region and the transverse 

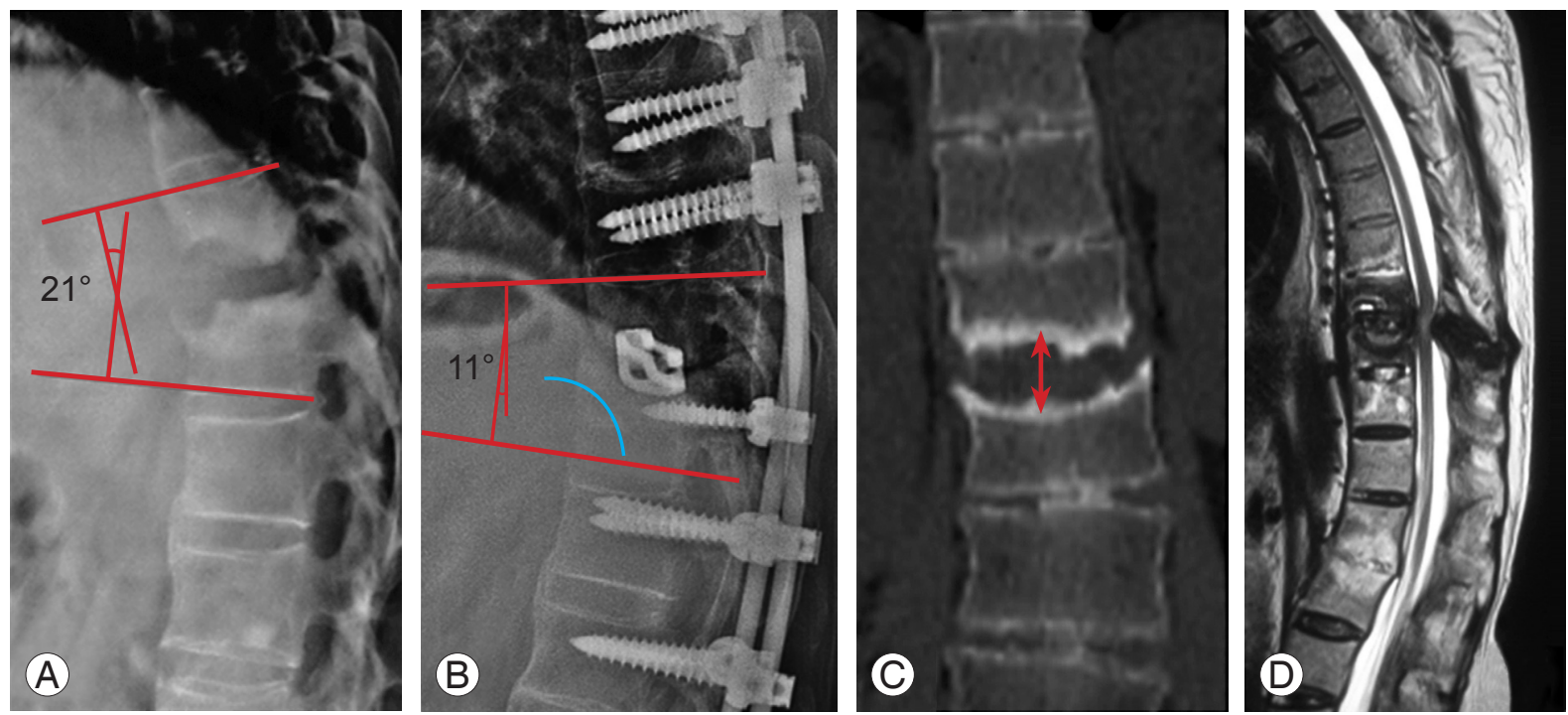

Fig. 1. Measurement of the regional kyphotic angle by Cobb's method in the (A) preoperative lateral radiograph, and (B) follow up lateral radiograph. (C) Assessment of the bony defect (shown with arrow) in the mid coronal section of computed tomography scan in the midvertebral level. (D) T2 weighted sagittal magnetic resonance imaging showing the pseudoarthrotic lesion causing compression of the spinal cord circumferentially.

processes in the lumbar region. Pedicle screw fixation was performed up to 3 or 4 levels above and below the lesion using the free hand technique. The extent of fixation was decided based on the presence of osteopenia, severity of kyphosis and the extent of anterior vertebral defect. After pedicle screw fixation, a temporary rod was placed unilaterally and a wide laminectomy was performed to remove the posterior fibrous pseudoarthrotic tissue and expose the exiting nerve roots laterally. After ligating the exiting nerve root, debridement of the anterior pseudoarthrotic lesion was performed through transforaminal, transpedicular or the lateral extra-cavitary approach depending on the location of the lesion. For lesions involving only the disc, the transforaminal approach was used whereas for lesions involving the vertebral and discovertebral regions, either the transpedicular (small defects) or the lateral extra-cavitary approach (large defects) was used (Fig. 2). Intraoperative tissue samples taken during debridement were processed for aerobic and anaerobic cultures. After adequate debridement and decompression, either local autograft or allograft was used to pack the anterior defect and an appropriate sized cage was tamped into the defect. In the lumbar region, care was taken to preserve the nerve roots and retract them to reach the anterior defect. After placing the cage, the affected spinal segment was compressed across the pedicle screws to secure the cage. Appropriately contoured rod was placed on

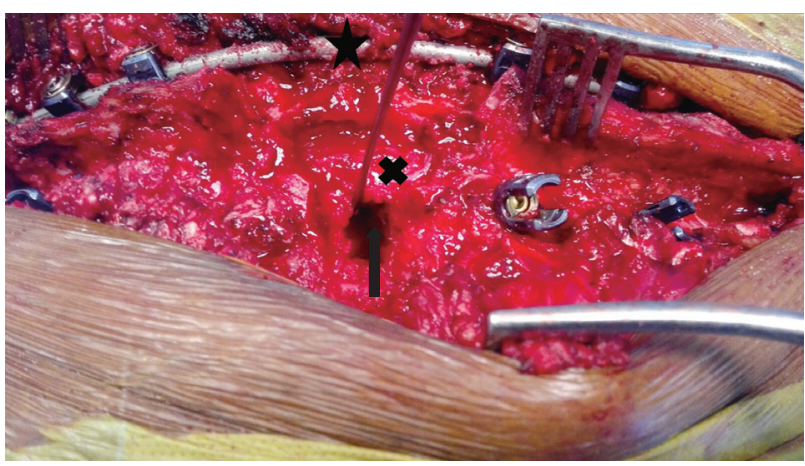

Fig. 2. Intraoperative clinical photograph showing the 'all-posterior' approach for stabilisation and anterior vertebral reconstruction. After a posterior approach, long segment pedicle screw fixation has been performed (the construct with rod fixed on the side contra lateral to the approach is shown by black cross mark). The spinal cord has been decompressed circumferentially. The anterior pseudoarthrosis has been addressed through the transforaminal approach shown by the black arrow.

the ipsilateral screws and compression performed across the spinal segment. Bone grafts were placed in the intertransverse region to promote circumferential fusion.

Postoperatively all the patients were mobilised with brace as early as the pain permitted. Patients were followed up at 6 weeks, and 3,6, and 12 months and then yearly if necessary. At follow up, all the patients were assessed radiologically for bony union using standard antero-posterior and lateral radiographs and functional 
outcomes were assessed by VAS, ASQoL, and ODI.

Data was analysed by using the SPSS ver. 17 (SPSS Inc., Chicago, IL, USA). Statistical significance was set at $p$-value $<0.05$ and the preoperative and postoperative outcome difference was assessed by paired $t$-test.

\section{Results}

There were a total of 20 patients of whom 18 were male and 2 were female. The mean age was 50.1 years (range, 26-74 years). The mean duration of symptoms was 17 months. The most common presenting symptom was persistent axial back pain $(n=16)$. Three patients presented with neurological deficit (1 ASIA C and 2 ASIA D) while one patient presented with progressive kyphosis. The preoperative VAS, ODI, and ASQoL were 8.2 $\pm 0.5,62.7 \pm 7.9$ and $14.3 \pm 2.08$, respectively. In the preoperative CT scan, the lesion was found to be involving all the three columns with a large anterior column defect of variable magnitude. The mean preoperative anterior column defect was $1.6 \pm 0.6 \mathrm{~cm}$.
The mean level of the posterior pedicle screw fixation was $6.2 \pm 2.4$ vertebrae. We had 8 patients who underwent of 3 level fixation proximal or distal to the defect. There were 12 patients who had 4 level fixation proximal or distal to the defect. The mean surgical duration, blood loss and LOS (length of stay) were 112 minuts, $452 \mathrm{~mL}$ and 6.2 days, respectively (Table 1). Intraoperative tissue samples sent for aerobic and anaerobic cultures did not isolate any organism in our series. Accidental dural tear occurred in 3 of the patients which were repaired and no postoperative CSF leak occurred. One patient had persistent headache and a CT scan of the brain revealed a pneumoencephalous which was treated conservatively without any sequalae. Postoperative hypoaesthesia in the dermatomal distribution of the sacrificed nerve root occurred in three patients but recovered eventually. One patient had a pleural tear during lateral extra-cavitary approach. It was not repaired and treated by inter-costal drainage. In the immediate postoperative period, 2 patients had incomplete neurological deficit which gradually improved to the

Table 1. Surgical details of the patients including blood loss, operative time, approach and bone graft used

\begin{tabular}{|c|c|c|c|c|c|c|}
\hline $\begin{array}{l}\text { Serial } \\
\text { number }\end{array}$ & $\begin{array}{l}\text { Blood loss } \\
\text { (mL) }\end{array}$ & $\begin{array}{l}\text { Operative } \\
\text { time (min) }\end{array}$ & $\begin{array}{c}\text { Approach to } \\
\text { anterior column }\end{array}$ & Bone graft & $\begin{array}{c}\text { Type of } \\
\text { titanium cage }\end{array}$ & $\begin{array}{c}\text { Preoperative WBC } \\
\text { count }\left(\mathrm{c} / \mathrm{mm}^{3}\right)\end{array}$ \\
\hline 1 & 310 & 95 & Transforaminal & Local autograft+allograft & Harms & 5,500 \\
\hline 2 & 250 & 95 & Transpedicular & Local autograft+allograft & Bullet & 6,300 \\
\hline 3 & 580 & 150 & Lateral extracavitory & Local autograft & Bullet & 8,200 \\
\hline 4 & 510 & 115 & Lateral extracavitory & Local autograft+allograft & Bullet & 5,600 \\
\hline 5 & 440 & 95 & Transforaminal & Local autograft+allograft & Bullet & 6,700 \\
\hline 6 & 750 & 145 & Lateral extracavitory & Local autograft & Bullet & 8,500 \\
\hline 7 & 250 & 95 & Transforaminal & Local autograft & Bullet & 8,000 \\
\hline 8 & 690 & 110 & Lateral extracavitory & Local autograft & Bullet & 7,500 \\
\hline 9 & 490 & 105 & Transforaminal & Local autograft & Bullet & 12,400 \\
\hline 10 & 410 & 95 & Transforaminal & Local autograft & Bullet & 9,600 \\
\hline 11 & 360 & 105 & Transforaminal & Local autograft & Bullet & 8,100 \\
\hline 12 & 260 & 85 & Transforaminal & Local autograft+allograft & Bullet & 12,300 \\
\hline 13 & 460 & 105 & Transforaminal & Local autograft & Bullet & 7,400 \\
\hline 14 & 470 & 95 & Transforaminal & Local autograft & Bullet & 10,000 \\
\hline 15 & 520 & 165 & Lateral extracavitory & Local autograft+allograft & Harms & 6,700 \\
\hline 16 & 730 & 135 & Lateral extracavitory & Local autograft+allograft & Harms & 8,200 \\
\hline 17 & 370 & 80 & Transpedicular & Local autograft & Bullet & 7,200 \\
\hline 18 & 610 & 165 & Lateral extracavitory & Local autograft & Bullet & 6,800 \\
\hline 19 & 230 & 115 & Transforaminal & Local autograft & Bullet & 8,800 \\
\hline 20 & 360 & 95 & Transforaminal & Local autograft & Bullet & 8,400 \\
\hline
\end{tabular}

WBC, white blood cells. 
preoperative level by the final follow up. Superficial surgical site infections occurred in 3 of the patients which were managed with oral antibiotics and serial regular dressings. No patient required a second surgical procedure.

The average follow-up was 2.1 years (range, 18 months4 years). Back pain was reduced in all the patients which was shown by the improvement in VAS from $8.2 \pm 0.5$ preoperatively to $2.4 \pm 0.6$ at the final follow-up $(p<0.05)$. ASQoL improved from $14.3 \pm 2.08$ to $7.90 \pm 1.48(p<0.05)$. ODI improved from $62.7 \pm 7.9$ to $18.5 \pm 7.5(p<0.05)$ (Table 2). Neurological improvement was seen in all the patients by at least one grade improvement in the ASIA scale with the two ASIA D patients recovering to ASIA E and ASIA C patient improving to ASIA D neurology at final follow up. None of the patients in our series were on any form of biologic drug therapy and were using only Non-steroidal anti-inflammatory drugs, Tramadol and Paracetamol for pain relief.

All patients had achieved radiological union at a mean $7.2 \pm 4.6$ months. Fusion was evident in the form of bridging bone across the defect in antero-posterior and lateral radiographs. At the final follow up, there was no recurrence of the lesion or progressive deformity or loss of correction and no implant failure occurred in any patient (Fig. 3). The mean preoperative regional kyphotic angle was $27^{\circ}$ which was corrected to $16.7^{\circ}$ postoperatively $(p<0.05)$, and maintained at $18.1^{\circ}$ at the final follow-up.

\section{Discussion}

Andersson lesions are lesions involving either one or both vertebral columns in patients with end stage ankylosing spondylitis. The prevalence varies between $1.5 \%$ and $28 \%$ in different studies [18-20]. This wide variation can be attributed to the varied presentation of these lesions masquerading as infection, inflammatory spondylitis and also due to lack of proper diagnostic criteria. Although AL in AS is well described, it is misdiagnosed commonly as infective discitis. In the series by Dave et al. [11], ten out of 29 patients had been misdiagnosed as tuberculosis and had taken anti-tubercular chemotherapy before surgical intervention. In our series, two patients were initially treated with anti-tubercular treatment before they presented to our institute. Successful management of AL depends on early diagnosis and appropriate intervention. Conservative treatment with brace, rest and physiotherapy is often the first offered mode of management in early lesions. Fang et al. [8] treated 27 patients of AL with bed rest, oral nonsteroidal anti-inflammatory drugs, and brace with good results in 14 patients. Thirteen patients with persistent pain eventually required surgical intervention. In general, there is paucity of studies on the predictors of successful outcome with conservative management in AL.

Surgery is the mainstay of management in patients with progressive kyphotic deformity, neurological deficit and
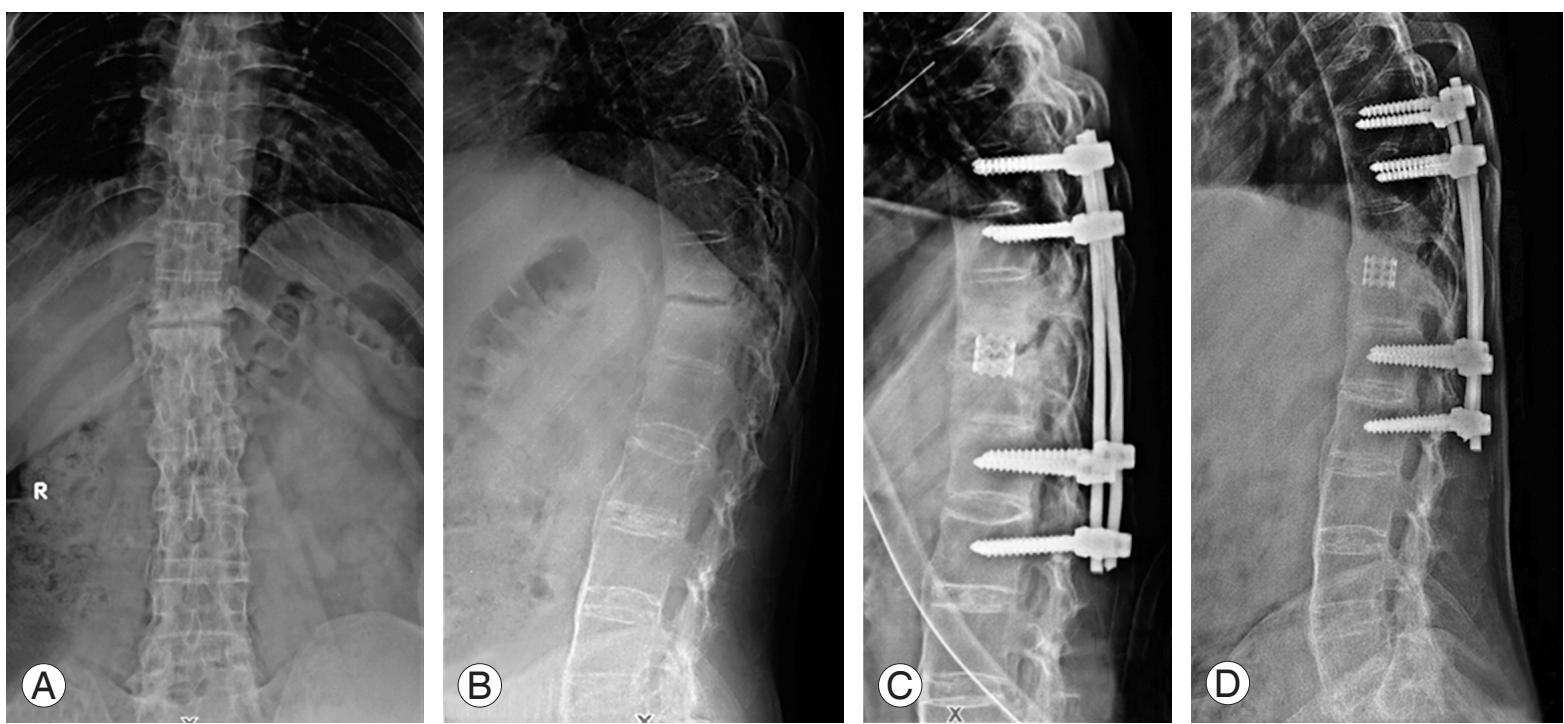

Fig. 3. A 26-year-old, diagnosed with ankylosing spondylitis 6 years back, presented with persistent back pain not responding to conservative management. Preoperative anteroposterior and lateral radiograph (A, B) shows at T11-12 pseudoarthrosis with kyphosis. (C) The lesion has been addressed by a long segment all-posterior approach stabilisation and posterior reconstruction. (D) The final follow-up lateral radiograph shows good union. 


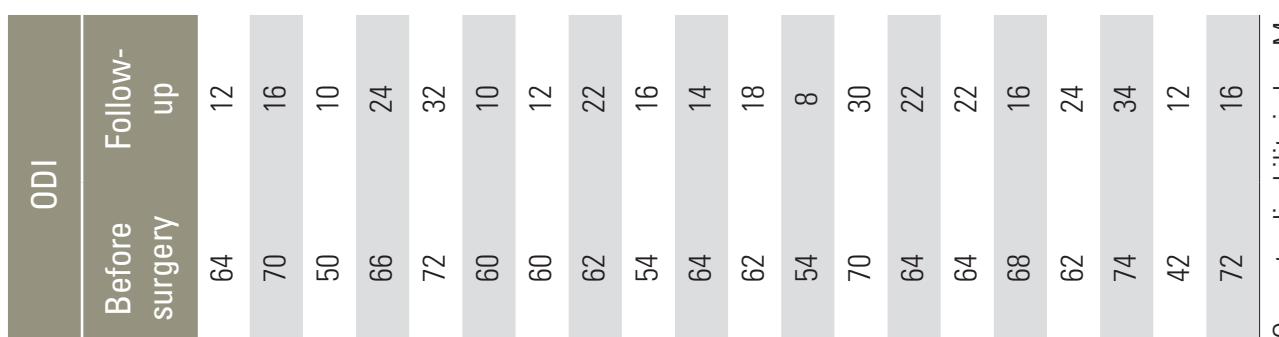

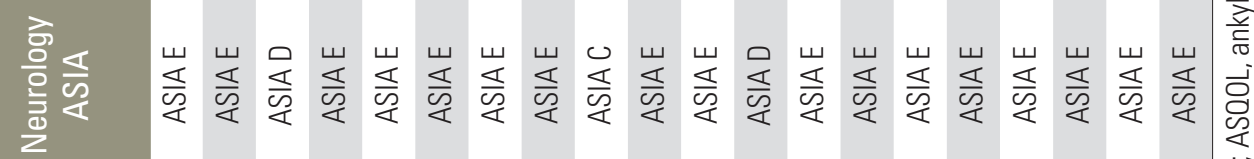

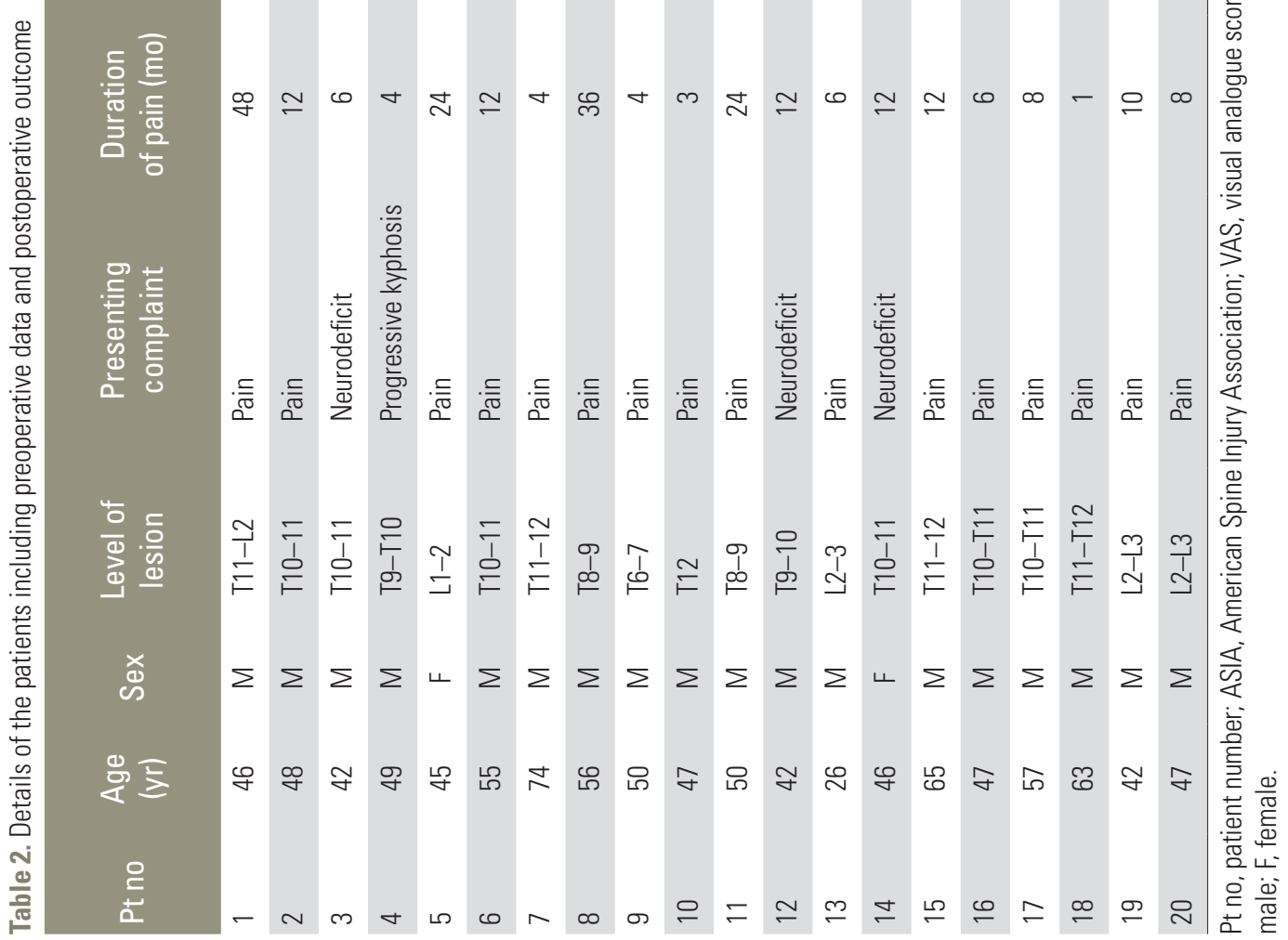


failure of adequate trial of conservative treatment. Many surgical methods have been described such as anterior surgery with fusion, combined anterior and posterior surgery, posterior alone surgery with or without correction of the anterior lesion and deformity, posterior single segment fixation with bone grafting, and posterior alone surgery with anterior debridement of the lesion [8-13,21,22]. However, till now no single surgical option can be considered ideal.

Fang et al. [8] studied 40 AL lesions in 35 patients over a period of 16 years. Surgery was performed in 16 patients for neurological deficit and failed conservative care. The patients underwent anterior debridement and reconstruction with autogenic iliac crest grafting or rib grafting within the anterior defect. The patients were confined to bed for two weeks and mobilised for six weeks of plaster jacket application. At a mean follow-up of 7.7 years, two patients had pseudoarthrosis and one patient required an additional posterior surgery. The radiological results of severity of defect and kyphosis have not been documented. Further ankylosing spondylitis is characterised by restricted lung function [17] and thus an anterior approach has the disadvantage of entering the thoracic cavity [17]. Since the biomechanics of AL requires a long segment fusion, an isolated anterior fixation in AL risks pseudoarthrosis resulting in persistent back pain and graft failure.

Kim et al. [10], performed a combined two staged posterior and anterior approach in AL. In the first stage, along with long segment posterior fixation, an osteotomy was performed at the level of AL (Smith Peterson osteotomy in 11 patients and Pedicle subtraction osteotomy in 6 patients), to achieve sagittal balance and correction of the deformity. Subsequently anterior surgery was performed to repair the pseudoarthrosis and bone grafting. The average correction of segmental kyphosis with SPO at the level of pseudoarthrosis was $20.9^{\circ}$ (range, $5^{\circ}-34^{\circ}$ ) and $26.3^{\circ}$ (range, $20^{\circ}-32^{\circ}$ ) with lumbar PSO. The mean sagittal imbalance had improved by $15.2 \mathrm{~cm}$ (range, $6.7-34.7 \mathrm{~cm}$ ) at the final follow-up. In our series, deformity correction was not attempted while the aim was to achieve stability and circumferential fusion. The mean preoperative regional kyphotic angle was $27^{\circ}$ at the level of pseudoarthrosis which was significantly corrected to $16.7^{\circ}$ postoperatively. The use of combined anterior and posterior surgeries is a major surgical undertaking in patients with ankylosing spondylitis. In contrast, Chang et al. [21] in 30 patients of AS with AL performed posterior only surgery with opening wedge osteotomy without any kind of anterior fusion. Despite leaving the anterior defect unattended, they showed good clinical and radiological results at a mean follow-up of 4.7 years in all their patients. However, the extent of fixation required to achieve healing and the mean anterior vertebral defect has not been described in their study. The authors propose that good bone healing capacity of AS can help in the healing of the pseudoarthrosis if adequate stabilisation is achieved by posterior instrumentation.

Wang et al. [22] had treated 8 patients with posterior only surgery with posterior instrumentation and posterolateral bone grafting without any debridement of the anterior lesion or any osteotomy, and showed good solid bony fusion. They proposed that minimal persistent motion at the pseudoarthrotic site as the reason for the non-union and posterior stabilisation alone without any anterior clearance achieves good fusion and effective pain relief. Recently, Zhang et al. [13] performed posterior only surgery with transpedicular subtraction and disc resection osteotomy and pedicle screw stabilisation in 17 consecutive patients with AL and showed satisfactory kyphotic correction with good bony union and favourable clinical outcomes. The average operating time was 219 minutes (range, 170-260 minutes) and the mean intra operative blood loss was $876 \mathrm{~mL}$, which was much higher than what we observed in our patient cohort. Wang et al. [12] in 12 patients showed that a posterior transforaminal or transpedicular approach, with debridement of the anterior lesion and bone graft and correction of the kyphotic deformity achieves good union, with improvement of the sagittal balance and better functional outcomes, and a supplemental anterior approach is unnecessary. In both the studies, the mean time to achieve anterior fusion has not been described.

Biomechanically, to effectively counteract the persistent motion at the pseudoarthrotic site adjacent long segments of fused vertebra, long segment fixation appears essential. To add complexity to the discussion, Dave et al. [11] studied 14 patients of AL treated only with posterior short segment pedicle screw fixation and bone grafting and showed no implant failures. However, they have not reported on the severity of anterior defect and the changes in kyphosis angle postoperatively, which are highly essential to decide on the efficacy of this procedure. Such a plethora of treatment options ranging from posterior short segment fixation alone to combined anterior and posterior surgery ex- 


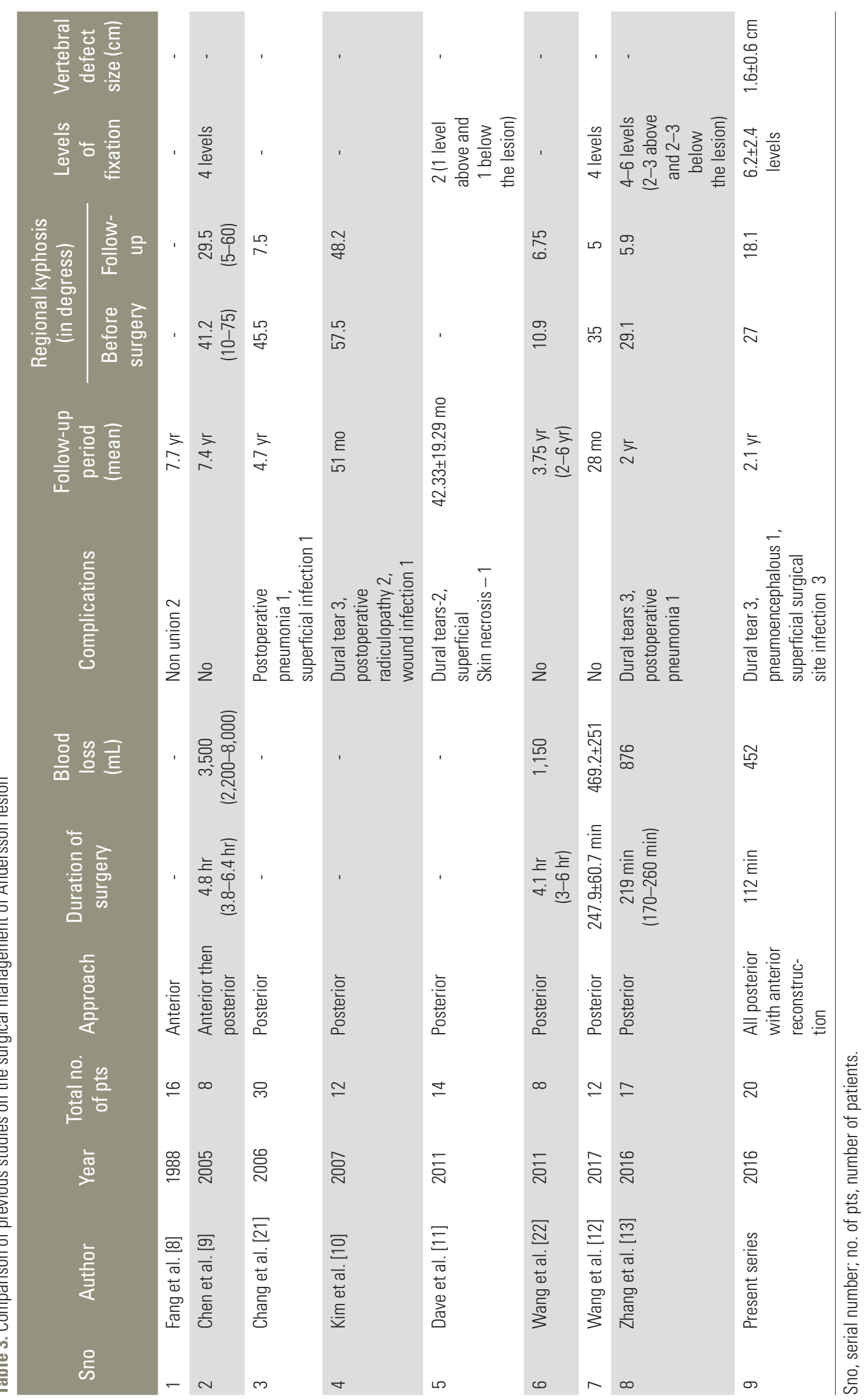


ist for AL (Table 3). But all the studies have shown similar clinical outcomes of good fusion and improved functional results.

None of the studies have reported major implant failure or postoperative neurological deficit. In our study, two patients had transient neurological worsening which improved by the final follow-up. Dural adhesions are found commonly in patients with AS and hence the chances of accidental dural tear are also higher as seen in our series and in other studies. The lack of protective epidural fat in the lesional area could be another reason for accidental dural injuries. The occurrence of pneumoencephalous has not been reported in other studies. One patient who also had a dural tear had persistent headache and upon evaluation with CT brain, he was diagnosed to have pneumoencepahalus. Though common after intracranial procedures, it is rare after spine procedures. It was treated conservatively with rest, analgesics and intravenous fluid supplementation.

The wide variations in the clinical and radiological parameters documented in previous studies and the small patient cohort in all the studies considering the rarity of the lesion restrains us from drawing strong conclusions from the existing studies. Most studies advise posterior surgery with long segment fixation because AL involves both columns of the spine and the altered biomechanics of the fused spine in AS can result in high stress on the implant. We believe that the presence of both anterior and posterior column disruption, wide anterior column defect (15 mm), significant kyphosis >20 degrees and vertebral osteopenia as seen in our study population, would better be treated by long segment fixation combined with anterior cage reconstruction. Long segment posterior fixation and anterior column reconstruction is considered to provide better stability with less chances of implant failure in a rigid, osteoporotic ankylosed spine in the presence of anterior column defect and kyphosis.

Limitations of this study include the small sample size and the retrospective nature of the study design. Though case controlled or randomized studies comparing the efficacy of one over the other technique would be ideal, the paucity of surgically treatable AL patients makes such studies not feasible.

\section{Conclusions}

Based on our results, we believe that posterior only sur- gery with long segment fixation and debridement of the lesion with anterior reconstruction can achieve circumferential solid fusion and good pain relief. Anterior reconstruction performed through transforaminal or lateral extracavitary approach is safe, feasible and enables good bony union and favourable clinical outcomes.

\section{Conflict of Interest}

No potential conflict of interest relevant to this article was reported.

\section{Acknowledgments}

The project was funded by Ganga Orthopaedic Research \& Education Foundation, Coimbatore.

\section{References}

1. Andersson O. Rontgenbilden vid spondylarthritis ankylopoetica. Nord Med Tidskr 1937;14:2002-4.

2. Rasker JJ, Prevo RL, Lanting PJ. Spondylodiscitis in ankylosing spondylitis, inflammation or trauma? A description of six cases. Scand J Rheumatol 1996;25: 52-7.

3. Nikolaisen C, Nossent H. Early histology in ankylosing spondylitis related spondylodiscitis supports its inflammatory origin. Scand J Rheumatol 2005;34:396-8.

4. Dihlmann W, Delling G. Disco-vertebral destructive lesions (So-called Andersson Lesions) associated with ankylosing spondylitis. Skeletal Radiol 1978;3: 10-6.

5. Wu PC, Fang D, Ho EK, Leong JC. The pathogenesis of extensive discovertebral destruction in ankylosing spondylitis. Clin Orthop Relat Res 1988;(230):15461.

6. Lohr KM, Barthelemy CR, Schwab JP, Haasler GB. Septic spondylodiscitis in ankylosing spondylitis. J Rheumatol 1987;14:616-20.

7. Bron JL, de Vries MK, Snieders MN, van der HorstBruinsma IE, van Royen BJ. Discovertebral (Andersson) lesions of the spine in ankylosing spondylitis revisited. Clin Rheumatol 2009;28:883-92.

8. Fang D, Leong JC, Ho EK, Chan FL, Chow SP. Spinal pseudarthrosis in ankylosing spondylitis. Clinicopathological correlation and the results of anterior 
spinal fusion. J Bone Joint Surg Br 1988;70:443-7.

9. Chen LH, Kao FC, Niu CC, Lai PL, Fu TS, Chen WJ. Surgical treatment of spinal pseudoarthrosis in ankylosing spondylitis. Chang Gung Med J 2005;28:621-8.

10. Kim KT, Lee SH, Suk KS, Lee JH, Im YJ. Spinal pseudarthrosis in advanced ankylosing spondylitis with sagittal plane deformity: clinical characteristics and outcome analysis. Spine (Phila Pa 1976) 2007; 32:1641-7.

11. Dave BR, Ram H, Krishnan A. Andersson lesion: are we misdiagnosing it? A retrospective study of clinicoradiological features and outcome of short segment fixation. Eur Spine J 2011;20:1503-9.

12. Wang T, Wang D, Cong Y, Yin C, Li S, Chen X. Evaluating a posterior approach for surgical treatment of thoracolumbar pseudarthrosis in ankylosing spondylitis. Clin Spine Surg 2017;30:E13-8.

13. Zhang X, Wang Y, Wu B, Hu W, Zhang Z, Wang Y. Treatment of Andersson lesion-complicating ankylosing spondylitis via transpedicular subtraction and disc resection osteotomy, a retrospective study. Eur Spine J 2016;25:2587-95.

14. Ikard RW. Methods and complications of anterior exposure of the thoracic and lumbar spine. Arch Surg 2006;141:1025-34.

15. Memtsoudis SG, Vougioukas VI, Ma Y, Gaber-Baylis LK, Girardi FP. Perioperative morbidity and mortality after anterior, posterior, and anterior/posterior spine fusion surgery. Spine (Phila Pa 1976) 2011;36:186777.

16. Carter S, Lories RJ. Osteoporosis: a paradox in ankylosing spondylitis. Curr Osteoporos Rep 2011;9:1125.

17. Ragnarsdottir M, Geirsson AJ, Gudbjornsson B. Rib cage motion in ankylosing spondylitis patients: a pilot study. Spine J 2008;8:505-9.

18. Kabasakal Y, Garrett SL, Calin A. The epidemiology of spondylodiscitis in ankylosing spondylitis: a controlled study. Br J Rheumatol 1996;35:660-3.

19. Cawley MI, Chalmers TM, Kellgren JH, Ball J. Destructive lesions of vertebral bodies in ankylosing spondylitis. Ann Rheum Dis 1972;31:345-58.

20. Chan FL, Ho EK, Fang D, Hsu LC, Leong JC, Ngan H. Spinal pseudarthrosis in ankylosing spondylitis. Acta Radiol 1987;28:383-8.

21. Chang KW, Tu MY, Huang HH, Chen HC, Chen YY, Lin CC. Posterior correction and fixation without anterior fusion for pseudoarthrosis with kyphotic deformity in ankylosing spondylitis. Spine (Phila Pa 1976) 2006;31:E408-13.

22. Wang G, Sun J, Jiang Z, Cui X. The surgical treatment of Andersson lesions associated with ankylosing spondylitis. Orthopedics 2011;34:e302-6. 